\title{
Challenge information logistics in decentralised structures.
}

\author{
H. Kühnle ${ }^{l)}$, K.-H. Sternemann ${ }^{2)}$ \\ ${ }^{1)}$ Otto-von-Guericke-Universität Magdeburg \\ Lehrstuhl für Fabrikbetrieb und Produktionssysteme \\ Universitätsplatz 2, 39106 Magdeburg \\ Tel: 03916718617 \\ Fax: 03916712404 \\ e-mail:kuehnle@iff.fhg.de \\ ${ }^{2)}$ University (TH) Karlsruhe, Institute for Machine Tools and \\ Production Science (wbk), Kaiserstrasse 12, D-76128 \\ Karlsruhe \\ Tel: $+49-0721-608-4010$ \\ Fax: +49-0721-699153 \\ e-mail: karlheinz.sternemann@mach.uni-karlsruhe.de
}

\begin{abstract}
The knowledge of the individual employee within a company is getting more and more important. It is therefore necessary to collect this knowledge more efficiently and to distribute this knowledge in such a way that more employees can take advantage of this information. This document describes a possibility how this knowledge could be used by everyone in a company through collecting the information, documents and relevant data and to safe them in a „Collaborative information space" in useful hyperlink structures. The most important part of the construction of a "Collaborative information space" in the sense of a shared context is the shared application, for production, collection and the definitions by uniformed categories and subjects. It is essential that this index contains a shared, polyhierarchical classification system which makes it easier to make use of this knowledge.
\end{abstract}

Keywords

information logistics, knowledge management, information management 


\section{INTRODUCTION}

Today the enterprises are confronted with higher requirements e.g. the integration of technical and logistical flow of work, or the organisational flexibility which requires complete different methods and tools for coping with the complexity involved. More and more enterprises create and have information which are produced manually or by a computer, which means in the same time a lack of support for decisions combined with an informational overload. It is for sure that it is much more difficult to control and to administrate the necessary information the bigger the database and more flexible the set-up and drain of the organisation are. The defined supply of information and knowledge is decisive for future business success. In times where information and knowledge becomes a central value added fact and competitiveness tool, the availability plays an important role apart from the quality of the commercial information. While the available electronically information has increased, the improvement of how to get the right information has stayed primitive.

\section{INFORMATION AND KNOWLEDGE AS A PROBLEM?}

The explosive growth of information due to reasonable priced mass storage systems and the fast communication networks have lead to the fact that the employees are not able anymore to make use of the information they are looking for. One main reason for the flood of information is the lack of quality and the distribution in the sense of "fitness for use" in real business processes. The information in an enterprise are often too extensive, too vague, too unstructured and cannot be interpreted properly. A study of Times/Sqirbe shows that the thousand biggest enterprises of Europe waste about DM 50 B yearly by searching information.

A solution could be the so called document-management-systems which are more and more often connected with information logistic and knowledge management. What is hidden behind these definition?

Information logistic is concerned with the distribution of information in the quality and quantity necessary, the right time, the right place, and cost. This means that information logistic should make it possible for an enterprise to get, produce, process, safe, distribute and use the information necessary. Without giving a definition of knowledge management, it means the collection, organisation and usage of knowledge under the consideration of learning processes within an enterprise. 


\section{How do we differ data, information and knowledge?}

Data seen for itself is a description of facts without a context. Information are drawn off data, transferred to communication processes and interpreted in the actual context. Therefore it is important to have knowledge. Knowledge is the information of possibilities of application of information in real processes. Knowledge is always connected with a person - contrary to data and information. The knowledge of the individual employee within a company is getting more and more important. It is therefore necessary to collect this knowledge more efficiently and to distribute this knowledge in such a way that more employees can take advantage of this information.

The standard situation of today is that many users still search with the help of search machines or information agents. The amount of search results, sometimes only one hyperlink but quiet often thousands links, found with help of this search machines still has to be analysed ,by hand“ to find the kind of information needed. The information found is sorted according to the relevance of the data, but many users interrupt this way of seeking information after a short time. It is the same with researches in databases.

To make it possible for an user to see if the information found is important for the current situation it is necessary to clear the context of the information early. Before an information can be used we have to know the requirements needed for the interpretation and the understanding of this information. At the same time information must be placed in a concrete situation, its origin context must be clear. This succeeds when it is shown in which way the information has to be used and in which situation it can be used.

It is necessary therefore

- To know who is the knowledge carrier for the current business process and the location of it.

- To see if other employees need this knowledge to.

- To place it in such a way that every employee can find the information easily.

- To find out what kind of knowledge is missing to improve a real business process step.

- To find a way to sort the information without getting an overload of them.

\section{PROVISION AND VISUALISATION OF INFORMATION}

In order to utilise hypermedia systems successfully in the technical environment of an enterprise, the particular context of an application in the enterprise must be considered. Predominant user requirements are:

- Integration of information and enterprise processes and

- A powerful navigation mechanism for information search

Communication between humans in the described context requires a physical medium for information storage and an interaction method. An appropriate information structure and intuitive mechanisms for navigation, are necessary for an efficient, goal oriented communication. To satisfy these requirements, the concept 
of a 'Collaborative Information Space' together with an elaborate navigation support is postulated [1].

\section{The Concept of the 'Collaborative Information Space'}

Starting from the mental layer of individual humans and their specific knowledge and perception, the 'Collaborative Information Space' shall enable a structured presentation of interpretable, cognitive pictures on a physical layer and store it in a common knowledge base. The described concept of the 'Collaborative Information Space' is built on the concept of 'semantic networks' introduced by Shannon and Weaver [2] and Nauta [3].

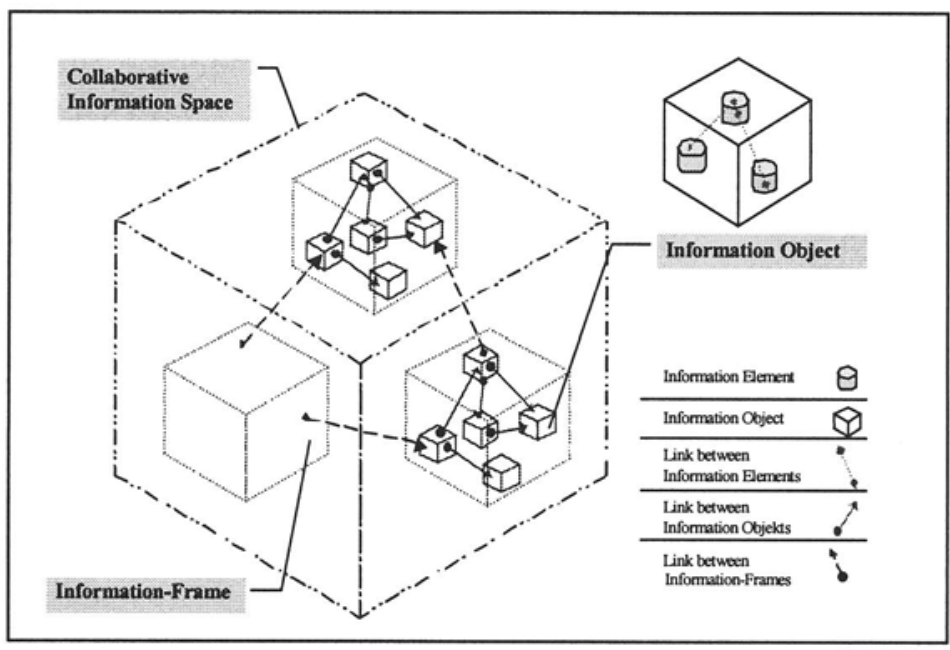

Figure 1: Structure and Components of the Collaborative Information Space.

Figure 1 shows the structure of the Collaborative Information Space as a three dimensional cube and its set of components. The necessary references to information entities, methods and relationships are defined in relations to the three dimensions, e.g. all information entities and their relationships are referenced, hence, the user can select his particular information object. Information does not fulfil a purpose in itself, but serves to support the value adding processes in the enterprise.

The most important part of the construction of a "Collaborative information space“ in the sense of a shared context is the shared application for producing, collecting and defining the information by uniformed categories and subjects. It is essential that this index of knowledge contains a shared, polyhierarchical classification system which makes it easier to make use of this knowledge.

The situation today is that information is classified into the type of information and the place where it is stored. This classification of information is not very useful for employees in enterprises - they do not or only little know the technical 
information- infrastructure and therefore information and knowledge are very difficult to reproduce.

A new way to differentiate information and knowledge is to create a ,structure space" with three primary structure dimensions.

- Process and activities

- Sphere of action, organisation- or functional areas

- Aspect of information, specific view or topic

The structure of the Collaborative Information Space corresponds to a classification scheme which defines three generic, top level object classes Processes, Function Domains and Information Aspects. The internal structure is governed by three dimensional information objects. Additional properties of objects such as object type or time can be added. The detail properties of objects are described in the Annex.

The mental perceptions of a human sender/provider are transformed, assembled and stored in the Collaborative Information Space into physical Information Objects. Information Objects should be specified in a catalogue of object classes, which represent the relevant enterprise information. Each element of a class provides an piece of information. The user receives the structured information needed for decision support, captures and interprets it in his own perception.

The structure is similar to hierarchies in a file system but exists independently of a file system. Data are stored and maintained only once in their original state. In the Collaborative Information Space, data are presented as Information Objects in a hierarchy structure. The hierarchy of Information Objects serves several purposes :

- The user can navigate in the three dimensions Processes, Function Domains and Information Aspect and the hierarchies of the Collaborative Information Space.

- The structure of the Collaborative Information Space enables the selection of information and relationships (hyperlinks)

- The user can search explicitly for one or several information objects or types The structure supports the allocation of access rights. Some users or user groups may only read information objects of a domain, while others have the additional right to change this information. 
In addition to these points additional ones can be integrated like object- or document type, object-owner or authorisations for access. Figure 2 clarifies the context and the interdependencies of this structure-dimension from the view of an employee. The concept of the "Collaborative information space" requires in addition to the information technology a systematic and interdisciplinary integration into the enterprise organisation.

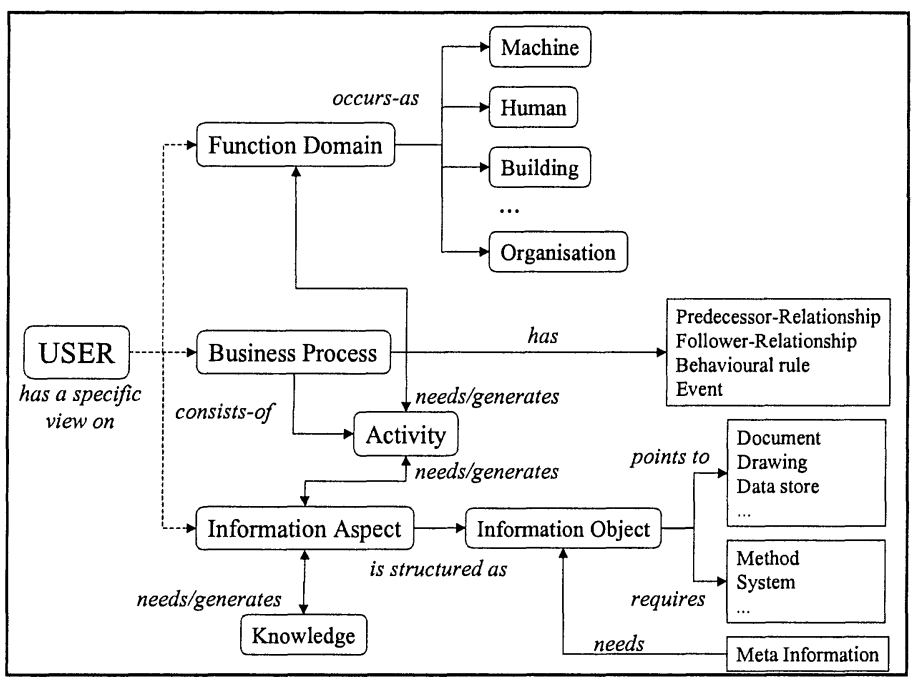

Figure 2: Semantic information model of the Collaborative Information Space.

Figure 3 clarifies this integration in connection to the 6 level model from Kühnle. It shows clearly, that social interaction between humans are influenced by cultural conditions in the enterprise and therefore the usage and the actuality of the whole common knowledge memory is influenced. 


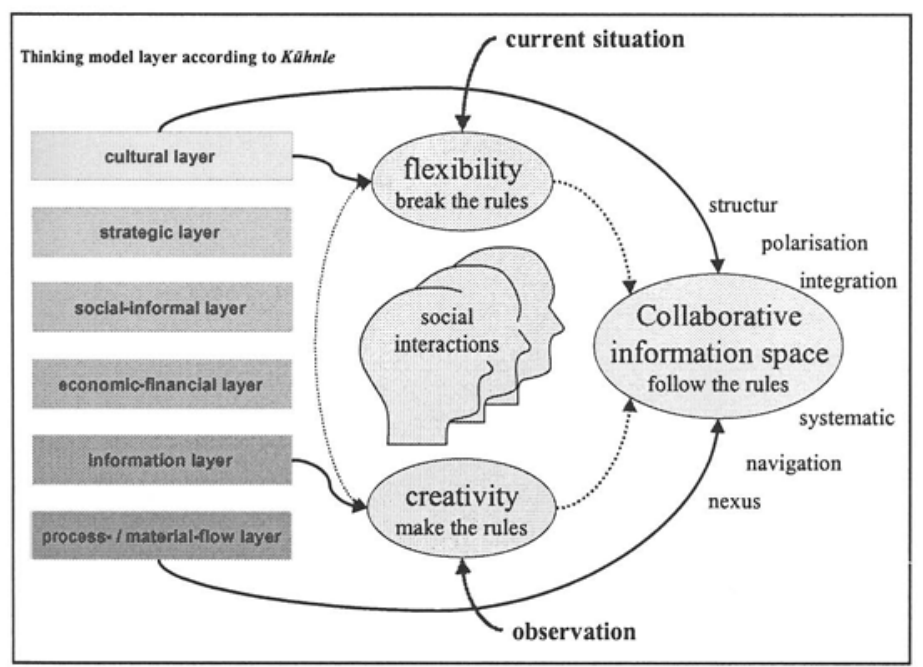

Figure 3: Social interaction model of the Collaborative Information Space.

\section{CONCLUSION}

The Collaborative Information Space - a structured space for information objects provides enterprise information to the user by matching information demand and supply based on the enterprise processes and can be employed as a tool for business (re-) engineering and for knowledge management. When operating on this information model, the user can navigate with different methods in the structure of the Collaborative Information Space. The methods support a navigation by creating semantic relationships between information objects. Several navigation options have been introduced and implemented in a prototype: Navigation with the Structure Browser, with block circuit diagrams or with drawings along the three dimensions of the Collaborative Information Space enable the user to find the desired information objects via different access paths. Making a search by the category object type provides an effective selection of the relevant information objects for a specific task out of a large data inventory.

The structure of the Collaborative Information Space enables a requirement oriented provision of information from heterogeneous and distributed inventories of data, information and knowledge. The Collaborative Information Space is using WEB technologies and, with an object broker, allows to access data bases, file systems and applications. With specified interfaces, distributed applications can employ methods or services in federated information systems and their environments supported by specific system integration functions. The business can operate this model based information system and utilise it for decision support. 


\section{REFERENCES}

[1] Kühnle, H.; Sternemann, K.-H.: Informations- und Wissensmanagement in dezentralen Strukturen; In: Kühnle, Sternemann, Harz (Hrsg): Herausforderung Geschäftsprozesse, Logis-Verlag, Stuttgart 1998

[2 Weaver, W.; Shannon, C.E. (Eds): 'The Mathematical Theory of Communication', University of Illinois, Chicago/London 1969

[3] Nauta, D. : 'The Meaning of Information', Mouton de Gruyter, The Hague, 1972

ANNEX : Properties of Information Object IO

Information Object IO is a multimedia information entity - text, drawings, sound, video - and represents the basic building block in the Collaborative Information Space CIS. IO receives structured information from the $C I S$. $I O$ has a label with a name and a reference. Any $I O$ can be aggregated to a larger $I O$. $I O$ represents a node in a hypertext system and its content is not limited to text but can include all types of multimedia entities- pictures, drawings, sound etc. In addition, methods or method calls - referenced to objects - are needed to generate the dynamical properties of $I O$.

Information Relationships IR

A set of $I O$ does not yet represent a $C I S$. Only the relationships between $I O$ lead to a semantic network and the introduction of functions within the overall context transforms a group of $I O$ into the $C I S$. Therefore, the basic element $I R$ is introduced. This relationship establishes dynamical connections to other information or physical objects or to method constructs.

A key property of the new concept is the equal treatment of $I O$ and $I R . I R$ is an object in itself and is stored like $I O$ in an adjacency-matrix. $I R$ is defined following previous concepts - with both a target anchor and a source anchor. However, this type of relationship is not sufficient to build coherency fields in the technical enterprise environment. Therefore, a second type of relationship is introduced. The attributes of $I R$ are extended, adding a structure parameter, which describes the structure dimensions of the CIS. 
Locating Information Objects and resources

A common query procedure for $I O$ and resources is an essential prerequisite. Because the potentials to optimise the enterprise operation and management can only be fulfilled by a natural intuitive utilisation of an information system. Until today, a standard query procedure for different information sources is not yet available. A similar problem had to be solved during the development of the internet. The technique to localise addresses can be applied to the intranet in the enterprise environment. For the Internet, the Uniform Resource Locator (URL) ${ }^{2}$ is used for the unambiguous location of resources. An URL is composed of the parts $<$ service $>: / /<$ host $>/ /<$ path $>\mid<$ file $>$. This principle is now extended with methods and accompanying parameters. Instead of a file, a method is called. A set of parameters is attached to the method, which enables an unambiguous identification of an object in the CIS.

\section{BIOGRAPHY}

Prof. Dr.-Ing.(habil), Dipl.Math. Hermann Kühnle was until 1994 director at the Fraunhofer-Institut für Produktionstechnik und Automatisierung Stuttgart. Since 1994 he is managing institute director of the Fraunhofer-Institut für Fabrikbetrieb und -automatisierung Magdeburg as well as director of the Institut für Arbeitswissenschaften, Fabrikautomatisierung und Fabrikbetrieb (IAF) at the Otto-von-Guericke Universität Magdeburg.

Dipl.-Ing. Karl-Heinz Sternemann is a member of the Institute of Machine Tools and Production Science (wbk) at the University Karlsruhe (TH). He was managing the research project DARIF within the framework concept 'production 2000' of the BMBF.

\footnotetext{
${ }^{2}$ Internet Standard RFC 1738 (December 1997)
} 